\title{
Algebraic Coset Conformal Field Theories II
}

By

Feng $\mathrm{XU}^{*}$

\begin{abstract}
Some mathematical questions relating to coset conformal field theories (CFT) are considered in the framework of algebraic quantum field theory as developed previously by us. We consider the issue of fix point resolution in the diagonal cosets of type A. We show how to decompose certain reducible representations into irreducibles, and prove that the coset CFT gives rise to a unitary modular category and therefore may be used to construct 3-manifold invariants. We prove that if the coset inclusion satisfies certain conditions which can be checked in examples, the Kac-Wakimoto Hypothesis (KWH) is equivalent to the Kac-Wakimoto Conjecture (KWC), a result which seems to be hard to prove by purely representation considerations. Examples are also presented.
\end{abstract}

\section{§1. Introduction}

This paper is a sequel to [X4]. Let us first recall some definitions from [X4].

Let $G$ be a simply connected compact Lie group and let $H \subset G$ be a connected Lie subgroup. Let $\pi^{i}$ be an irreducible representations of $L G$ with positive energy at level ${ }^{1} k$ on Hilbert space $H^{i}$ (cf. §2.1). Suppose when restricting to $L H, H^{i}$ decomposes as:

$$
H^{i}=\sum_{\alpha} H_{i, \alpha} \otimes H_{\alpha}
$$

and $\pi_{\alpha}$ are irreducible representations of $L H$ on Hilbert space $H_{\alpha}$. The set of $(i, \alpha)$ which appears in the above decompositions will be denoted by

Communicated by T. Miwa, April 6, 1999. Revised September 13, 1999.

1991 Mathematics Subject Classification(s): 46S99, 81R10

* Department of Mathematics, University of Oklahoma, 423 Physical Science Center Norman, Oklahoma 73019, USA.

I'd like to thank Professors J. Fuchs, K-H. Rehren and C. Dong for useful correspondences. This work is partially supported by the NSF under Grant DMS 9820935.

1 When $G$ is the direct product of simple groups, $\dot{k}$ is a multi-index, i.e., $k=\left(k_{1}, \ldots, k_{n}\right)$, where $k_{i} \in N$ corresponding to the level of the $i$-th simple group. The level of $L H$ is determined by the Dynkin indices of $H \subset G$. To save some writing we write the coset as $H \subset G_{k}$. 
exp.

We shall use $\pi^{1}$ (resp. $\left.\pi_{1}\right)^{2}$ to denote the vacuum representation of $L G$ (resp. $L H$ ). Let $\mathscr{A}$ be the vacuum sector of the coset $G / H$ as defined in $\S 2.1$ of [X4]. The decompositions above naturally give rise to a class of covariant representations of $\mathscr{A}$, denoted by $\pi_{i, \alpha}$ or simply $(i, \alpha)$. By Th. 2.3 of [X4], $\pi_{1,1}$ is the vacuum representation of $\mathscr{A}$.

In $\S 2.2$ we consider the decompositions of certain reducible representations in the diagonal cosets of type $A_{N-1}$ as considered in $\$ 4.3$ of [X4] when the action of the Dynkin diagram automorphisms is not faithful (cf. (2) of Th. 4.3 of [X4]), which is part of the fixed point resolution problems known in physics literature (cf. [Gep], [LVW] and [SY]). Such problems have been known for some time, and there are no even clear mathematical formulations of such questions before. We will show that the results of $[\mathrm{X} 4]$ provide the right mathematical framework for understanding such questions.

We first prove a general Lemma 2.1 which we believe will play an important role in all fixed point resolution problems. Using Lemma 2.1 and Lemma 2.2, we prove (cf. (1) of Th. 2.3) that certain $S$-matrices are non-degenerate. It follows from Th. 2.3 (Cor. 2.4) that the diagonal cosets of type $A_{N-1}$ give rise to a unitary modular category in the sense of Turaev (cf. P. 74 and P. 113 of $[\mathrm{Tu}]$ ), and may be used to construct 3-manifold invariants (cf. P. 160 of [Tu]). We also calculate $S$ matrices when $N$ is prime. The results agree with some of the results of [FSS1], [SY] from different considerations based on physics.

To describe the results in $\S 3$, let us denote by $S_{i j}$ (resp. $S_{\alpha \beta}^{\circ}$ ) the $S$ matrices of $L G$ (resp. $L H$ ) at level $k$ (resp. certain level of $L H$ determined by the inclusion $H \subset G_{k}$ ). Define $^{3}$

$$
b(i, \alpha)=\sum_{(j, \beta)} S_{i j} \vec{S}_{\alpha \beta}\langle(j, \beta),(1.1)\rangle
$$

where $\langle$,$\rangle is defined in \S 2.1$. Note the above summation is effectively over those $(j, \beta)$ such that $(j, \beta) \in \exp$. The Kac-Wakimoto Conjecture (KWC) states

2 This is slightly different from the notation $\pi^{0}$ (resp. $\pi_{0}$ ) in [X4]: it seems to be more appropriate since these representations correspond to identity sectors.

${ }^{3}$ Our $(j, \beta)$ corresponds to $(M, \mu)$ on P. 186 of $[\mathrm{KW}]$, and it follows from the definitions that $\langle(j, \beta),(1,1)\rangle$ is then equal to mult $_{M}(\mu, p)$ which appears in 2.5 .4 of $[\mathrm{KW}]$. Our formula (1) is then identical to 2.5 .4 of $[\mathrm{KW}]$. 
that if $(i, \alpha) \in \exp$, then $b(i, \alpha)>0$.

The Kac-Wakimoto Hypothesis (KWH) states that if $\langle(j, \beta),(1,1)\rangle>0$ and $(i, \alpha) \in \exp$, then $S_{i j} \vec{S}_{\alpha \beta} \geq 0$.

Note that since $S_{i 1}>0, \dot{S}_{\alpha 1}>0,(1,1) \in \exp , \mathrm{KWH}$ implies KWC.

$\mathrm{KWC}$ has proved to be true in all known examples. In fact, in $\S 2$ of [X4] an even stronger conjecture, Conjecture 2(C2) is formulated (also cf. [L4]).

Unfortunately $\mathrm{KWH}$ is not true. In [X2] counter examples were found by using subfactors associated with conformal inclusions. However, KWH has been checked to be true in so many examples, and it seems that it should be true or equivalent to $\mathrm{KWC}$ under some general conditions. The first main result in $\S 3.1$ is to describe such a condition (cf. Th. 3.3). The condition ${ }^{4}$ is that:

$$
\text { if }\langle(1, \beta),(1,1)\rangle>0 \text {, then } \beta=1 \text {. }
$$

Th. 3.3 states that if $H \subset G_{k}$ satisfies (2), and certain assumptions in $\S 3.1$ which are expected to be true in general, then $\mathrm{KWH}$ is equivalent to $\mathrm{KWC}$ for the inclusion $H \subset G_{k}$.

Condition (2) can be shown to be equivalent to the normality of certain inclusions, but we will not discuss this in this paper.

In $\S 3.1$ we also give an example which does not satisfy (2), and verifies $\mathrm{KWC}$ but not $\mathrm{KWH}$. This is also the first example of non-conformal inclusion which does not verify $\mathrm{KWH}$.

It is interesting to note that Th. 3.3 can be thought as a statement about representations of affine Kac-Moody algebras without even mentioning von Neumann algebras, yet it seems to be hard to obtain such results without using subfactor theory (cf. [J]). We give another example of this nature in Prop. 3.2. For more such statements, see inequality on P. 11 of [X2] and in particular (2) of Th. 4.3 of [X4].

In $\S 3.2$ we prove a property (Prop. 3.4) of Conjecture 2 (C2) in [X4]. It states that if $H_{1} \subset H_{2}$ and $H_{2} \subset G$ verify $\mathrm{C} 2$, then $H_{1} \subset G$ also verifies $\mathrm{C} 2$, thus reducing $\mathrm{C} 2$ to maximal inclusions which are classified in [Dyn1], [Dyn2]. We also give an example related to $N=2$ superconformal theories.

\footnotetext{
${ }^{4}$ If we identify $(1, \beta)$ with $(M, \mu)$, where $M$ is the vacuum representation, as on P. 186 of [KW], then condition (2) is the statement that if $(M, \mu) \in S_{m}$, with $S_{m}$ defined on P. 186 of [KW], then $\mu$ must be the vacuum representation of the subalgebra.
} 
§2. Fixed Point Resolutions in the Diagonal Cosets of Type $A_{N-1}$

§2.1 Preliminaries. Let us first recall some definitions from [X2]. Let $M$ be a properly infinite factor and $\operatorname{End}(M)$ the semigroup of unit preserving endomorphisms of $M$. In this paper $M$ will always be the unique hyperfinite $I I I_{1}$ factors. Let $\operatorname{Sect}(M)$ denote the quotient of $\operatorname{End}(M)$ modulo unitary equivalence in $M$. We denote by $[\rho]$ the image of $\rho \in \operatorname{End}(M)$ in $\operatorname{Sect}(M)$.

It follows from [L3] and [L4] that $\operatorname{Sect}(M)$, with $M$ a properly infinite von Neumann algebra, is endowed with a natural involution $\theta \rightarrow \bar{\theta}$; moreover, $\operatorname{Sect}(M)$ is a semiring with identity denoted by id.

If given a normal faithful conditional expectation $\epsilon: M \rightarrow \rho(M)$, we define a number $d_{\epsilon}$ (possibly $\infty$ ) by:

$$
d_{\epsilon}^{-2}:=\operatorname{Max}\left\{\lambda \in[0,+\infty) \mid \epsilon\left(m_{+}\right) \geq \lambda m_{+}, \forall m_{+} \in M_{+}\right\}
$$

(cf. $[P P])$.

We define

$$
d=\operatorname{Min}_{\epsilon}\left\{d_{\epsilon} \mid d_{\epsilon}<\infty\right\}
$$

$d$ is called the statistical dimension of $\rho$. It is clear from the definition that the statistical dimension of $\rho$ depends only on the unitary equivalence classes of $\rho$. The properties of the statistical dimension can be found in [L1], [L2] and [L3]. We will denote the statistical dimension of $\rho$ by $d_{\rho}$ in the following. $\quad d_{\rho}^{2}$ is called the minimal index of $\rho$.

Recall from [X2] that we denote by $\operatorname{Sect}_{0}(M)$ those elements $\operatorname{of} \operatorname{Sect}(M)$ with finite statistical dimensions. For $\lambda, \mu \in \operatorname{Sect}(M)$, let $\operatorname{Hom}(\lambda, \mu) \subset M$ denote the space of intertwiners from $\lambda$ to $\mu$, i.e. $a \in \operatorname{Hom}(\lambda, \mu)$ iff $a \lambda(x)=\mu(x) a$ for any $x \in M$. $\operatorname{Hom}(\lambda, \mu)$ is a finite dimensional vector space and we use $\langle\lambda, \mu\rangle$ to denote the dimension of this space. $\langle\lambda, \mu\rangle$ depends only on [ $\lambda]$ and $[\mu]$. Moreover we have $\langle v \lambda, \mu\rangle=\langle\lambda, \bar{v} \mu\rangle,\langle v \lambda, \mu\rangle=\langle v, \mu \bar{\lambda}\rangle$ which follows from Frobenius duality (See [L2]). We will also use the following notation: if $\mu$ is a subsector of $\mu$, we will write as $\mu \prec \lambda$ or $\lambda \succ \mu$. A sector is said to be irreducible if it has only one subsector.

Recall (cf. [L2]) of each $\rho \in \operatorname{End}(M)$ and its conjugate $\bar{\rho}$ with finite minimal index, there exists $R_{\rho} \in \operatorname{Hom}(i d, \bar{\rho} \rho)$ and $\bar{R}_{\rho} \in \operatorname{Hom}(i d, \rho \bar{\rho})$ such that

$$
\bar{R}_{\rho}^{*} \rho\left(R_{\rho}\right)=1, R_{\rho}^{*} \bar{\rho}\left(\bar{R}_{\rho}\right)=1
$$

and $\left\|R_{\rho}\right\|=\left\|\bar{R}_{\rho}\right\|=\sqrt{d_{\rho}}$. The minimal left inverse $\phi_{\rho}$ of $\rho$ is defined by 


$$
\phi_{\rho}(m)=R_{\rho}^{*} \bar{\rho}(m) R_{\rho} .
$$

The following lemma plays a fundamental role in $\S 2.2$.

Lemma 2.1. Let $a, b, c \in \operatorname{End}(M),[c]=[a b]$ and $a, b$ have finite statistical dimensions. Suppose $\tau \in \operatorname{End}(M)$ has order $t$ in $\operatorname{Sect}(M)$, i.e., $t$ is the least positive integer such that $\left[\tau^{t}\right]=[i d]$, and $[a \tau]=[a],[\tau b]=[b]$. If

$$
\langle c, c\rangle=t
$$

then $\operatorname{Hom}(c, c)$ is an abelian algebra with dimension $t$ and hence there exist irreducible sectors $c_{1}, \ldots, c_{t}$ such that

$$
c=\sum_{1 \leq k \leq t} c_{k}
$$

Moreover, $d_{c_{k}}=\frac{1}{t} d_{c}, k=1, \ldots, t$

Proof. From $[a \tau]=[a]$ we conclude by using Frobenius duality that $\left\langle\bar{a} a, \tau^{i}\right\rangle \geq 1$, and since $\tau$ has order $t$ in $\operatorname{Sect}(M)$, we must have

$$
\bar{a} a>\sum_{0 \leq i \leq t-1} \tau^{i}
$$

Similarly

$$
b \bar{b}>\sum_{0 \leq i \leq t-1} \tau^{i}
$$

Since

$$
\begin{aligned}
t=\langle c, c\rangle & =\langle a b, a b\rangle=\langle\bar{a} a, b \bar{b}\rangle \\
& \geq \sum_{0 \leq i \leq t-1}\left\langle\bar{a} a, \tau^{i}\right\rangle\left\langle\tau^{i}, b \bar{b}\right\rangle \geq t,
\end{aligned}
$$

it follows that all the $\geq$ are $=$ and in particular $\langle\bar{a} a, i d\rangle=\langle\bar{b} b, i d\rangle=1$, i.e., both $a$ and $b$ are irreducible. It is enough to prove the case $c=a b$. Since $[\tau b]=[b]$, there exist unitary elements $v \in M$ such that

$$
v \in \operatorname{Hom}(b, \tau b) \text {. }
$$

Define $\tau_{v}=v^{*} \tau v$, then 


$$
b=\tau_{v} b
$$

and so

$$
b=\tau_{v}^{t} b
$$

Since $\left[\tau^{t}\right]=[i d]$, there exists a unitary $v_{1}$ such that $\tau_{v}^{t}=A d_{v_{1}}$, where $A d_{v_{1}}(m):=v_{1} m v_{1}^{*}, \forall m \in M$. So $v_{1} \in \operatorname{Hom}(b, b)$. Since $b$ is irreducible, $v_{1}$ is equal to identity up to a complex number whose absolute value is 1 , so

$$
\tau_{v}^{t}=i d
$$

From $[a \tau]=\left[a \tau_{v}\right]=[a]$ there exists a unitary $u$ such that $u \in \mathbb{H o m}\left(a \tau_{v}, a\right)$. It follows that

$$
u^{t} a(m)=u^{t} a\left(\tau_{v}^{t}(m)\right)=a(m) u^{t}, \forall m \in M
$$

so $u^{t}=x .1$ with $x \in \mathbb{C}, x$ has absolute value one, since $a$ is irreducible. Define $w=x^{\frac{1}{t}} u$ so that $w^{t}=1$. Note $w \in \operatorname{Hom}(a b, a b)$ since $b=\tau_{v} b$. Denote by $\phi_{a}, \phi_{b}$ the minimal left inverses of $a, b$ respectively. We claim that $\phi_{b} \phi_{a}\left(w^{i}\right)=d_{a} d_{b} \delta_{i 0}$ for $0 \leq i \leq t-1$. It is enough to show $\phi_{b} \phi_{a}\left(w^{i}\right)=0$ for $0<i \leq t-1$. Since $b$ is irreducible, $\phi_{a}\left(w^{i}\right) \in \operatorname{Hom}(b, b) \equiv \mathbb{C} .1$, and so $\phi_{b} \phi_{a}\left(w^{i}\right)=R_{b}^{*} \phi_{a}\left(w^{i}\right) R_{\bar{b}}$. But

$$
\begin{aligned}
R_{b}^{*} \phi_{a}\left(w^{i}\right) R_{\bar{b}} & =R_{b}^{*} R_{a}^{*} \bar{a}\left(w^{i}\right) R_{a} R_{\bar{b}}=R_{a}^{*} \bar{a} a\left(R_{b}^{*}\right) \bar{a}\left(w^{i}\right) R_{a} R_{\bar{b}} \\
& =R_{a}^{*} \bar{a}\left(a\left(R_{\bar{b}}^{*}\right) w^{i}\right) R_{a} R_{\bar{b}}=R_{a}^{*} \bar{a}\left(w^{i} a \tau_{v}^{i}\left(R_{\bar{b}}^{*}\right)\right) R_{a} R_{\bar{b}} \\
& =R_{a}^{*} \bar{a}\left(w^{i}\right) \bar{a} a\left(\tau_{v}^{i}\left(R_{b}^{*}\right)\right) R_{a} R_{\bar{b}} \\
& =R_{a}^{*} \bar{a}\left(w^{i}\right) R_{a} \tau_{v}^{i}\left(R_{b}^{*}\right) R_{\bar{b}},
\end{aligned}
$$

and use the fact $\tau_{v} b=b$ we have $\tau_{v}^{i}\left(R_{b}^{*}\right) R_{\bar{b}} \in \operatorname{Hom}(b, b)\left(i d, \tau_{v}^{i}\right)$. Since $\left\langle\tau^{i}, i d\right\rangle$ $=0,0<i \leq t-1$, it follows that $\tau_{v}^{i}\left(R_{b}^{*}\right) R_{\bar{b}}=0$ and so $\phi_{b} \phi_{a}\left(w^{i}\right)=d_{a} d_{b} \delta_{i 0}$ for $0 \leq i \leq t-1$.

If $\Sigma_{0 \leq i \leq t-1} x_{i} w^{i}=0, \quad x_{i} \in \mathbb{C}$, multiply both sides by $w^{t-i}$ and apply $\phi_{b} \phi_{a}$, we get $x_{i}=0$. So $i d, w, w^{2}, \cdots, w^{t-1}$ are linearily independent in $\operatorname{Hom}(c, c)$. And since $\operatorname{Hom}(c, c)$ has dimension $t, \operatorname{Hom}(c, c)$ is therefore an abelian algebra with basis $i d, w, w^{2}, \cdots, w^{t-1}$. Note $w^{t}=1$, so the minimal projections $\mathbb{P}_{k}, k=1, \cdots, t$ in $\operatorname{Hom}(c, c)$ are given by $\mathbb{P}_{k}=\Sigma_{0 \leq j \leq t-1} \frac{1}{t}\left(\exp \left(\frac{2 \pi i k}{t}\right) w\right)^{j}$. Let $c_{k} \prec c$ be the irreducible sector corresponding to $P_{k}$, then by Th. 5.5 of [L1]

$$
d_{c_{k}}=\phi_{a} \phi_{b}\left(P_{k}\right)=\frac{1}{t} d_{a} d_{b}=\frac{1}{t} d_{c}, k=1, \cdots, t . \quad \text { Q.E.D. }
$$


Next we will recall some of the results of [Reh] (also cf. [FRS]) and introduce notations.

Let $\left\{\left[\rho_{i}\right], i \in I\right\}$ be a finite set of equivalence classes of irreducible superselection sectors (cf. [GL]). Suppose this set is closed under conjugation and composition. We will denote the conjugate of $\left[\rho_{i}\right]$ by $\left[\rho_{i}\right]$ and identity sector by [1] if no confusion arises, and let $N_{i j}^{k}=\left\langle\left[\rho_{i}\right]\left[\rho_{j}\right],\left[\rho_{k}\right]\right\rangle$. We will denote by $\left\{T_{e}\right\}$ a basis of isometries in $\operatorname{Hom}\left(\rho_{k}, \rho_{i} \rho_{j}\right)$. The univalence of $\rho_{i}$ (cf. P. 12 of $[\mathrm{GL}]$ ) will be denoted by $\omega_{\rho_{i}}$.

Let $\phi_{i}$ be the unique minimal left inverse of $\rho_{i}$, define:

$$
Y_{i j}:=d_{\rho_{i}} d_{\rho_{j}} \phi_{j}\left(\epsilon\left(\rho_{j}, \rho_{i}\right) \epsilon\left(\rho_{i}, \rho_{j}\right)\right)^{*},
$$

where $\epsilon\left(\rho_{j}, \rho_{i}\right)$ is the unitary braiding operator (cf. [GL]).

We list two properties of $Y_{i j}$ (cf. (5.13), (5.14) of [Reh]) which will be used in $\$ 2.2$ :

$$
\begin{gathered}
Y_{i j}=Y_{j i}=Y_{i j}^{*}=Y_{\overline{i j}} \\
Y_{i j}=\sum_{k} N_{i j}^{k} \frac{\omega_{i} \omega_{j}}{\omega_{k}} d_{\rho_{k}}
\end{gathered}
$$

Let us explain the proof of (2) since similar but different proof appears in the proof of lemma 2.2 .

We have:

$$
\begin{aligned}
\phi_{j}\left(\sum_{e} T_{e} T_{e}^{*} \epsilon\left(\rho_{i}, \rho_{j}\right)^{*} \epsilon\left(\rho_{j}, \rho_{i}\right)^{*} T_{e} T_{e}^{*}\right) & =\sum_{e} \frac{\omega_{i} \omega_{j}}{\omega_{k}} \phi_{j}\left(T_{e} T_{e}^{*}\right) \\
& =\sum_{e} \frac{\omega_{i} \omega_{j}}{\omega_{k}} N_{i j}^{k} \frac{d_{\rho_{k}}}{d_{\rho_{i}} d_{\rho_{j}}}
\end{aligned}
$$

where in the first $=$ we used the monodromy equation (cf. [FRS] or P. 359 [X1]), and the second $=$ follows from [L3]. (2) now follows immediately.

Define $\tilde{\sigma}:=\Sigma_{i} d_{\rho_{\mathrm{r}}}^{2} \omega_{\rho_{\mathrm{l}}}^{-1}$. If the matrix $\left(Y_{i j}\right)$ is invertible, by the proposition on P. 351 of $[\operatorname{Reh}] \tilde{\sigma}$ satisfies $|\tilde{\sigma}|^{2}=\Sigma_{i} d_{\rho_{i}}^{2}$. Suppose $\tilde{\sigma}=|\tilde{\sigma}| \exp (i x), x \in \mathbb{R}$. Define matrices

$$
S:=|\tilde{\sigma}|^{-1} Y, T:=\exp \left(i \frac{x}{3}\right) \operatorname{Diag}\left(\omega_{\rho_{\mathrm{v}}}\right)
$$


Then these matrices satisfy the algebra:

$$
\begin{gathered}
S S^{\dagger}=T T^{\dagger}=i d, \\
T S T S T=S, \\
S^{2}=C, T C=C T=T,
\end{gathered}
$$

where $C_{i j}=\delta_{i \bar{j}}$ is the conjugation matrix. Moreover

$$
N_{i j}^{k}=\sum_{m} \frac{S_{i m} S_{j m} S_{k m}^{*}}{S_{1 m}}
$$

(7) is known as Verlinde formula.

Now let us consider an example which verifies (1) to (7) above as in $\$ 1$ of [X1]. Let $G=S U(N)$. We denote $L G$ the group of smooth maps $f: S^{1} \mapsto G$ under pointwise multiplication. The diffeomorphism group of the circle $\operatorname{Diff} S^{1}$ is naturally a subgroup of $\operatorname{Aut}(L G)$ with the action given by reparametrization. In particular the group of rotations $\operatorname{Rot} S^{1} \simeq U(1)$ acts on $L G$. We will be interested in the projective unitary representation $\pi: L G \rightarrow U(H)$ that are both irreducible and have positive energy. This means that $\pi$ should extend to $L G \ltimes \operatorname{Rot} S^{1}$ so that $H=\oplus_{n \geq 0} H(n)$, where the $H(n)$ are the eigenspace for the action of $\operatorname{Rot} S^{1}$, i.e., $r_{\theta} \xi=\exp (\operatorname{in} \theta) \xi$ for $\xi \in H(n)$ and $\operatorname{dim} H(n)<\infty$ with $H(0) \neq 0$. It follows from [PS] that for fixed level $K$ which is positive integer, there are only finite number of such irreducible representations indexed by the finite set

$$
P_{++}^{h}=\left\{\lambda \in P \mid \lambda=\sum_{i=1, \cdots, N-1} \lambda_{i} \Lambda_{i}, \lambda_{i} \geq 1, \sum_{i=1, \cdots, N-1} \lambda_{i}<h\right\}
$$

where $P$ is the weight lattice of $S U(N)$ and $\Lambda_{i}$ are the fundamental weights and $h=N+K$. We will use 1 to denote the trivial representation of $S U(N)$. For $\lambda, \mu, v \in P_{++}^{h}$, define

$$
N_{\lambda \mu}^{v}=\sum_{\delta \in P_{+}^{n}+} \frac{S_{\lambda \delta} S_{\mu \delta} S_{v \delta}^{*}}{S_{1 \delta}}
$$

where $S_{\lambda \delta}$ is given by the Kac-Peterson formula: 


$$
S_{\lambda \delta}=c \sum_{w \in S_{N}} \varepsilon_{w} \exp (i w(\delta) \cdot \lambda 2 \pi / n)
$$

Here $\varepsilon_{w}=\operatorname{det}(w)$ and $c$ is a normalization constant fixed by the requirement that $\left(S_{\lambda \delta}\right)$ is an orthonormal system. It is shown in [Kac] P. 288 that $N_{\lambda \mu}^{v}$ are nonnegative integers. Moreover, define $\operatorname{Gr}\left(C_{K}\right)$ to be the ring whose basis are elements of $P_{++}^{h}$ with structure constants $N_{\lambda \mu}^{v}$. The natural involution $*$ on $P_{++}^{h}$ is defined by $\lambda \mapsto \lambda^{*}=$ the conjugate of $\lambda$ as representation of $S U(N)$. All the irreducible representations of $\operatorname{Gr}\left(C_{K}\right)$ are given by $\lambda \rightarrow \frac{S_{\lambda \mu}}{S_{1 \mu}}$ for some $\mu$.

The irreducible positive energy representations of $L S U(N)$ at level $K$ give rise to an irreducible conformal precosheaf $\mathscr{A}$ and its covariant representations (cf. P. 362 of [X1]). The unitary equivalent classes of such representations are the superselection sectors. We will use $\lambda$ to denote such representations.

For $\lambda$ irreducible, the univalence $\omega_{\lambda}$ is given by an explicit formula. Let us first define

$$
\Delta_{\lambda}=\frac{c_{2}(\lambda)}{K+N}
$$

where $c_{2}(\lambda)$ is the value of Casimir operator on representation of $S U(N)$ labeled by dominant weight $\lambda$ (cf. 1.4 .1 of [KW]). $\Delta_{\lambda}$ is usually called the conformal dimension, and $\omega_{\lambda}=\exp \left(2 \pi i \Delta_{\lambda}\right)$.

Define the central charge (cf. 1.4.2 of $[\mathrm{KW}]$ )

$$
C_{G}:=\frac{K \operatorname{dim}(G)}{K+N}
$$

and $T$ matrix as

$$
T=\operatorname{diag}\left(\dot{\omega}_{\lambda}\right)
$$

where $\dot{\omega}_{\lambda}=\omega_{\lambda} \exp \left(\frac{-2 \pi i C_{G}}{24}\right) . \quad$ By Th. 13.8 of $[\mathrm{Kac}] S$ matrix as defined in (9) and $T$ matrix in (12) satisfy relation (4), (5) and (6).

By Cor. 1 in $\S 34$ of [W], the fusion ring generated by all $\lambda \in P_{++}^{h}$ is isomorphic to $\operatorname{Gr}\left(C_{K}\right)$, with structure constants $N_{\lambda \mu}^{v}$ as defined in (8). One may therefore ask what are the $Y$ matrix (cf. (0)) in this case. By using (2) and the formula for $N_{\lambda \mu}^{v}$, a simple calculation shows:

$$
Y_{\lambda_{\mu}}=\frac{S_{\lambda_{\mu}}}{S_{1_{\mu}}},
$$


and it follows that $Y_{\lambda \mu}$ is nondegenerate, and $S, T$ matrices as defined in (3) are indeed the same $S, T$ matrix defined in (8) and (11), which is a surprising fact. If the analogue of Cor. 1 in $\S 34$ of [W] is established for other types of simple and simply connected Lie groups, then this fact is also true for other types of groups by the same argument.

In $\S 2.2$ we will also consider the case when $G$ is the direct product of two type $A$ groups. In that case the $S, T$ matrices are just the tensor product of the $S, T$ matrices corresponding to each subgroup.

\$2.2 Fixed point resolutions. We preserve the set up of $\S 4.3$ of $[\mathrm{X} 4]$. We consider the coset $G:=S U(N)_{m^{\prime}} \times S U(N)_{m^{\prime \prime}} / H:=S U(N)_{m^{\prime}+m^{\prime \prime}}$, where the embedding $H \subset G$ is diagonal. Let $\Lambda_{1}, \ldots, \Lambda_{N-1}$ be the fundamental weights of $S L(N)$. Let $k \in N$. Recall that the set of integrable weights of the affine algebra $\widehat{S L(N)}$ at level $k$ is the following subset of the weight lattice of $\operatorname{SL}(N)$ :

$$
P_{++}^{h}=\left\{\lambda=\lambda_{1} \Lambda_{1}+\cdots+\lambda_{N-1} \Lambda_{N-1} \mid \lambda_{i} \in \mathbb{N}, \lambda_{1}+\cdots+\lambda_{N-1}<h\right\}
$$

where $h=k+N$. This set admits a $\mathbb{Z}_{N}$ automorphism generated by

$$
\sigma_{1}: \lambda=\left(\lambda_{1}, \lambda_{2}, \ldots, \lambda_{N-1}\right) \rightarrow \sigma_{1}(\lambda)=\left(h-\sum_{j=1}^{N-1} \lambda_{j}, \lambda_{1}, \ldots, \lambda_{N-2}\right) .
$$

We define the color $\tau(\lambda): \equiv \Sigma_{i}\left(\lambda_{i}-1\right) \operatorname{imod}(N)$ and $Q$ to be the root lattice of $\widehat{S L(N)}$ (cf. $\S 1.3$ of $[\mathrm{KW}]$ ). Note that $\lambda \in Q$ iff $\frac{1}{N} \tau(\lambda) \in \mathbb{Z}$.

We use $i$ (resp. $\alpha)$ to denote the irreducible positive energy representations of $L G$ (resp. $L H)$. To compare our notations with that $\$ 2.7$ od $[\mathrm{KW}$, note that our $i$ is $\left(\Lambda^{\prime}, \Lambda^{\prime \prime}\right)$ of $[\mathrm{KW}]$, and our $\alpha$ is $\Lambda$ of $[\mathrm{KW}]$. We will identify $i=\left(\Lambda^{\prime}, \Lambda^{\prime \prime}\right)$ and $\alpha=\Lambda$ where $\Lambda^{\prime}, \Lambda^{\prime \prime}, \Lambda$ are the weights of $S L(N)$ at levels $m^{\prime}$, $m^{\prime \prime}, m^{\prime}+m^{\prime \prime}$ respectively. Suppose

$$
i=\left(\Lambda_{1}^{\prime}, \Lambda_{1}^{\prime \prime}\right), j=\left(\Lambda_{2}^{\prime}, \Lambda_{2}^{\prime \prime}\right), k=\left(\Lambda_{3}^{\prime}, \Lambda_{3}^{\prime \prime}\right), \alpha=\Lambda_{1}, \beta=\Lambda_{2}, \delta=\Lambda_{3} .
$$

Then the fusion coefficients $N_{i j}^{k}:=N_{\Lambda_{1}{ }^{\prime} \Lambda_{2}{ }^{\prime}}^{\Lambda^{\prime}} N_{\Lambda_{1}{ }^{\prime \prime}{ }_{2}{ }^{\prime \prime}}^{\Lambda^{\prime \prime}}\left(\right.$ resp. $\left.N_{\alpha \beta}^{\delta}:=N_{\Lambda_{1} \Lambda_{2}}^{\Lambda_{3}}\right)$ of $L G$ (resp. $L H)$ are given by Verlinde formula (cf. §2.1). Recall from $\S 4.3$ of [X4] that $\pi_{i, \alpha}$ are the covariant representations of the coset $G / H$. The set of all $(i, \alpha):=\left(\Lambda^{\prime}, \Lambda^{\prime \prime}, \Lambda\right)$ which appears in the decompositions of $\pi^{i}$ of $L G$ with respect to $L H$ is denoted by exp. This set is determined on P. 194 of [KW] to be $\left(\Lambda^{\prime}, \Lambda^{\prime \prime}, \Lambda\right) \in \exp$ iff $\Lambda^{\prime}+\Lambda^{\prime \prime}-\Lambda \in Q$. The $\mathbb{Z}_{N}$ action on $(i, \alpha), \forall i, \forall \alpha$ is denoted 
by $\sigma(i, \alpha):=\left(\sigma\left(\Lambda^{\prime}\right), \sigma\left(\Lambda^{\prime \prime}\right), \sigma(\Lambda)\right), \sigma \in \mathbb{Z}_{N}$. This is also known as diagram automorphisms since they corresponds to the automorphisms of Dykin diagrams. Note that this $Z_{N}$ action preserves $\exp$ and therefore induces a $\mathbb{Z}_{N}$ action on exp.

We define a vector space $W$ over $C$ whose orthonormal basis are denoted by $i \otimes \alpha$ with $i=\left(\Lambda^{\prime}, \Lambda^{\prime \prime}\right), \alpha=\Lambda$. W is also a commutative ring with structure constants given by $N_{i j}^{k} N_{\alpha \beta}^{\delta}$. Let $V$ be the vector space over $C$ whose basis are given by the irreducible components of $\sigma_{i} a_{1 \otimes \bar{\alpha}}$ (cf. $\S 4.3$ of [X4]). Then $V=V_{0} \oplus V_{1}$, where $V_{0}$ is a subspace of $V$ whose basis are given by the irreducible components of $\sigma_{i} a_{1 \otimes \bar{\alpha}}$ with $(i, \alpha) \in \exp$, and $V_{1}$ is the orthogonal complement of $V_{0}$ in $V$. The composition of sectors gives $V$ a ring structure. By (1) of Th. 4.3 of [X4], the irreducible subrepresentations of $(i, \alpha)$ of the coset are in one-to-one correspondence with the basis of $V_{0}$ and this map is a ring isomorphism by (1) of Prop. 4.2 of [X4], and we will identify the irreducible subrepresentations of $(i, \alpha)$ of the coset with the basis of $V_{0}$ in the following when no confusion arises. Note that $V_{0}$ is a subring of $V$ and $V_{0} . V_{1} \subset V_{1}$.

Define a linear map $P: W \rightarrow V$ such that $P(i \otimes \alpha)=\sigma_{i} a_{1 \otimes \bar{\alpha}} . \quad$ By Th. 4.3 of [X4]

$$
P(i \otimes \alpha)=\sigma_{i} a_{1 \otimes \bar{\alpha}}=P\left(i^{\prime} \otimes \bar{\alpha}^{\prime}\right)=\sigma_{i^{\prime}} a_{1 \otimes \alpha^{\prime}}
$$

iff $\sigma^{s}(i)=i^{\prime}, \sigma^{s}(\alpha)=\alpha^{\prime}$ for some $s \in \mathbb{Z}$. Also $\langle P(i \otimes \alpha), P(j \otimes \beta)\rangle=0$ if $P(i \otimes \alpha)$ $\neq P(j \otimes \beta)$ by $(*)$ of $\S 4.3$ of $[\mathrm{X} 4]$.

Note $P(\sigma(1) \otimes \sigma(1))=1$ and $P$ is a ring homomorphism from $W$ to $V$. Define $W_{0}:=P^{-1}\left(V_{0}\right), W_{1}:=P^{-1}\left(V_{1}\right)$, then $W=W_{0} \oplus W_{1}$ since $\exp$ is $\sigma$ invariant. Note that $i \otimes \alpha \in W_{0}$ iff $i-\alpha \in Q$. Define the action of $Z_{N}$ on $W$ as $\sigma(i \otimes \alpha)=\sigma(i) \otimes \sigma(\alpha)$. Much of the following depends on the relation between $W$ and $V$.

Assume $\sigma^{s}(i \otimes \alpha)=i \otimes \alpha$ for some $i \otimes \alpha \in W$, and $0<s \leq N$ is the least positive integer with this property. Let $t=\frac{N}{s}$. By equation $(*)$ in $\S 4.3$ of [X4] we have:

$$
\langle P(i \otimes \alpha), P(i \otimes \alpha)\rangle=t .
$$

Our first question is to decompose $P(i \otimes \alpha)$ when $t>1$.

Apply Lemma 2.1 to the present case with

$$
P(i \otimes 1)=a, P(1 \otimes \alpha)=b, P(i \otimes \alpha)=c, P\left(\sigma^{s} \otimes 1\right)=\tau,
$$

we conclude that there exists $c_{1}, \cdots, c_{t} \in V$ such that 


$$
P(i \otimes \alpha)=\sum_{1 \leq k \leq t} c_{k}
$$

and $d_{c_{k}}=\frac{1}{t} d_{i} d_{\alpha}, k=1, \ldots, t$. Note that if $P^{-1}(P(i \otimes \alpha))=\left\{i_{1} \otimes \alpha_{1}, \ldots, i_{s} \otimes \alpha_{s}\right\}$, then $s t=N$.

Note we identify the covariant representations of the coset with the basis of $P\left(W_{0}\right)=V_{0}$. The univalence of $A:=P(i \otimes \alpha), i \otimes \alpha \in W_{0}$ are given by: $\omega_{A}=\exp \left(2 \pi i\left(\Delta_{i}-\Delta_{\alpha}\right)\right)$, where $\Delta_{i}, \Delta_{\alpha}$ are the conformal dimensions (cf. $\S 2.1$, and if $\left.i=\left(\Lambda^{\prime}, \Lambda^{\prime \prime}\right), \Delta_{i}:=\Delta_{\Lambda^{\prime}}+\Delta_{\Lambda^{\prime \prime}}\right)$. Note if $A>a$, then $\omega_{a}=\omega_{A}$. The univalence is only defined for covariant sectors which correspond to elements of $V_{0}$. However, for convenience let us define $\omega_{i \otimes \alpha}:=\omega_{i} \omega_{\alpha}^{-1}$ for $i \otimes \alpha \in W$. Then if $i \otimes \alpha \in W_{0}, \omega_{i} \omega_{\alpha}^{-1}$ which is the univalence of $P(i \otimes \alpha)$ depends only on $P(i \otimes \alpha)$, i.e. . $\omega_{i} \omega_{\alpha}^{-1}$ depends only on the orbit of $i \otimes \alpha$ under the $Z_{N}$ action.

Suppose $A:=P(i \otimes \alpha), B:=P(j \otimes \beta), i \otimes \alpha, j \otimes \beta \in W_{0}$. Let $\phi_{A}, \phi_{B}$ be the unique minimal left inverses of $A, B$. Define

$$
Y_{A B}:=d_{A} d_{B} \phi_{B}\left(\phi_{A}(\epsilon(B, A) \epsilon(A, B))^{*}\right) .
$$

Note (1) is similar to (0) of $\S 2.1$, the difference here is that our $A, B$ may be reducible and hence we need to include $\phi_{B}$ in the definition since $\phi_{A}(\epsilon(B, A) \epsilon(A, B))^{*}$ may not be a scalar.

To avoid confusions we will denote $S$ matrices associated to indices $i$ (Recall from $\$ 2.1$ this is the tensor product of $S$ matrices associated to two type A subgroup of $G$ ) by $S_{i j}$ and the $S$ matrices associated to indices $\alpha$ by $S_{\alpha \beta}^{\cdot}$.

Lemma 2.2. Suppose $A:=P(i \otimes \alpha), B:=P(j \otimes \beta), i \otimes \alpha, j \otimes \beta \in W_{0}$, and $A$ $=\Sigma_{1 \leq i \leq t} c_{i}$ with $d_{c_{i}}=\frac{1}{t} d_{A}$. Then:

(1) $\left\langle c_{i} B, P(k \otimes \delta)\right\rangle=\frac{1}{t}\langle A B, P(k \otimes \delta)\rangle, \forall k \otimes \delta \in W$;

(2) $Y_{A B}=\frac{S_{i j}}{S_{11}} \frac{\dot{S}_{\alpha \beta}}{\dot{S}_{11}}$;

(3) $Y_{c_{i} B}=\frac{1}{t} Y_{A B}$;

(4) If $B=\Sigma_{j} b_{j}$, then $\Sigma_{j} Y_{c_{i} b_{j}}=Y_{c_{i} B}$.

Proof. Ad(1): Denote by $C:=P(k \otimes \delta)$. Then by Frobenius duality

$$
\left\langle c_{i} B, C\right\rangle=\left\langle c_{i}, C \bar{B}\right\rangle .
$$

By the definitions of $B, C$, 


$$
C \bar{B}=l A+\sum D
$$

where $l \geq 0$ is an integer, and $D$ are elements of the form $P\left(k^{\prime} \otimes \delta^{\prime}\right)$ which are different from $A$, and by $(*)$ in $\S 4.3$ of $[\mathrm{X} 4]\langle D, A\rangle=0$, and so $\left\langle D, c_{i}\right\rangle=0$. It follows that

$$
\left\langle c_{i} B, C\right\rangle=\left\langle c_{i}, l A\right\rangle=l
$$

which is independent of $i$. Since $A=\Sigma_{1 \leq i \leq t} c_{i}$, (1) follows.

Ad (2): The main point of the proof is that even though $A, B$ may be reducible, their univalence are complex numbers, so the monodromy equation (cf. [FRS] or P. 359 of [X1]) holds, and we have:

$$
\begin{aligned}
\phi_{B}\left(\phi_{A}(\epsilon(B, A) \epsilon(A, B))^{*}\right) & =\phi_{B}\left(\phi_{A}\left(\sum_{e \in V} T_{e} T_{e}^{*} \epsilon(A, B)^{*} \epsilon(B, A)^{*} T_{e} T_{e}^{*}\right)\right) \\
& =\sum_{e \in V} \frac{\omega_{A} \omega_{B}}{\omega_{e}} N_{A B}^{e} \frac{d_{e}}{d_{A} d_{B}} \\
& =\sum_{e \in V} \frac{\omega_{i} \omega_{j} \omega_{\alpha}^{-1} \omega_{\beta}^{-1}}{\omega_{e}}\langle A B, e\rangle \frac{d_{e}}{d_{A} d_{B}}
\end{aligned}
$$

where $e \in V$ means we sum over the basis of $V$. Note that the summation above is effectively over $V_{0}$ since $A \in V_{0}, B \in V_{0}$ and $V_{0}$ is a subring. Suppose $P(k \otimes \delta)=e_{1}+e_{2}+\cdots+e_{m}, k \otimes \delta \in W_{0}$, with $d_{e_{i}}=\frac{1}{m} d_{k} d_{\delta}, \omega_{e_{i}}=\omega_{k} \omega_{\delta}^{-1}$. Assume $P^{-1}(P(k \otimes \delta))=k_{1} \otimes \delta_{1}, \cdots, k_{n} \otimes \delta_{n}$, with $m n=N$. Then

$$
\sum_{e_{i}} \frac{\omega_{i} \omega_{j} \omega_{\alpha}^{-1} \omega_{\beta}^{-1}}{\omega_{e_{i}}}\left\langle A B, e_{i}\right\rangle \frac{d_{e_{i}}}{d_{A} d_{B}}=\frac{1}{m} \frac{\omega_{i} \omega_{j} \omega_{\alpha}^{-1} \omega_{\beta}^{-1}}{\omega_{k} \omega_{\delta}^{-1}}\langle A B, P(k \otimes \delta)\rangle \frac{d_{k} d_{\delta}}{d_{A} d_{B}} .
$$

By equation $(*)$ in $\S 4.3$ of $[\mathrm{X} 4]$,

$$
\begin{aligned}
\langle A B, P(k \otimes \delta)\rangle & =\left\langle\sigma_{i} a_{1 \otimes \bar{\alpha}_{j}} \sigma_{j} a_{1 \otimes \bar{\beta}}, \sigma_{k} a_{1 \otimes \bar{\delta}}\right\rangle=N_{i j}^{k^{\prime}} N_{\bar{\alpha} \bar{\beta}}^{\bar{\delta}_{\bar{\beta}}^{\prime}}\left\langle\sigma_{k^{\prime}} a_{1 \otimes \bar{\delta}^{\prime}}, \sigma_{k} a_{1 \otimes \bar{\delta}}\right\rangle \\
& =m\left(N_{i j}^{k_{1}} N_{\bar{\alpha} \overline{\bar{\beta}}}^{\bar{\delta}_{\bar{\beta}}}+\cdots+N_{i j}^{k_{n}} N_{\bar{\alpha}} \overline{\bar{\alpha}}_{\bar{\beta}}\right)
\end{aligned}
$$

We conclude that

$$
\sum_{e_{i}} \frac{\omega_{i} \omega_{j} \omega_{\alpha}^{-1} \omega_{\beta}^{-1}}{\omega_{e}}\left\langle A B, e_{i}\right\rangle \frac{d_{e_{i}}}{d_{A} d_{B}}=\frac{\omega_{i} \omega_{j} \omega_{\alpha}^{-1} \omega_{\beta}^{-1}}{\omega_{k} \omega_{\delta}^{-1}}\left(N_{i j}^{k_{1}} N_{\bar{\alpha} \bar{\beta}}^{\bar{\delta}_{1}}+\cdots+N_{i j}^{k_{n}} N_{\bar{\alpha} \overline{\bar{\beta}}}\right) \frac{d_{k} d_{\delta}}{d_{A} d_{B}},
$$


and so

$$
\begin{aligned}
\left.\phi_{B}\left(\phi_{A}(\epsilon(B, A) \epsilon A, B)\right)^{*}\right) & =\sum_{k \otimes \delta \in W} \frac{\omega_{i} \omega_{j} \omega_{\bar{\alpha}}^{-1} \omega_{\bar{\beta}}^{-1}}{\omega_{k} \omega_{\bar{\delta}}^{-1}} N_{i j}^{k} N_{\bar{\alpha} \bar{\beta}}^{\overline{\bar{\beta}}} \frac{d_{k} d_{\delta}}{d_{i} d_{\alpha} d_{j} d_{\beta}} \\
& =\sum_{k} \frac{\omega_{i} \omega_{j}}{\omega_{k}} N_{i j}^{k} \frac{d_{k}}{d_{i} d_{j}} \times \sum_{\delta} \frac{\omega_{\alpha}^{-1} \omega_{\beta}^{-1}}{\omega_{\delta}^{-1}} N_{\alpha \beta}^{\delta} \frac{d_{\delta}}{d_{\alpha} d_{\beta}} \\
& =\frac{S_{i j}}{S_{11}} \frac{\vec{S}_{\alpha \beta}}{\dot{S}_{11}}
\end{aligned}
$$

where in the last $=$ we have used (2) and the comment after (12) in $\$ 2.1$.

Ad (3): As in the proof of (2) let $P(k \otimes \delta), k \otimes \delta \in W_{0}, P(k \otimes \delta)=e_{1}+\cdots+e_{m}$, $P^{-1}(P(k \otimes \delta))=\left\{k_{1} \otimes \delta_{1}, \ldots, k_{n} \otimes \delta_{n}\right\}$, with $m n=N$. Then

$$
\begin{aligned}
\sum_{e_{j}}\left\langle c_{i} B, e_{j}\right\rangle \frac{\omega_{c_{i}} \omega_{B}}{\omega_{e_{j}}} d_{e_{j}} & =\frac{1}{m}\left\langle c_{i} B, P(k \otimes \delta)\right\rangle \frac{\omega_{A} \omega_{B}}{\omega_{k \otimes \delta}} d_{k} d_{\delta} \\
& =\frac{1}{t} \frac{1}{m}\langle A B, P(k \otimes \delta)\rangle \frac{\omega_{A} \omega_{B}}{\omega_{k \otimes \delta}} d_{k} d_{\delta} \\
& =\frac{1}{t} \sum_{e_{j}}\left\langle A B, e_{j}\right\rangle \frac{\omega_{A} \omega_{B}}{\omega_{e_{j}}} d_{e_{j}}
\end{aligned}
$$

where on the second = we used (1), and (3) follows immediately.

Ad (4): First note $\omega_{b_{j}}=\omega_{B}$. By (2) of $\S 2.1$, we have:

$$
\begin{aligned}
\sum_{j} Y_{c_{i} b_{j}} & =\sum_{j} \sum_{e}\left\langle c_{i} b_{j}, e\right\rangle \frac{\omega_{c_{i}} \omega_{b_{j}}}{\omega_{e}} d_{e}=\sum_{e} \sum_{j}\left\langle c_{i} b_{j}, e\right\rangle \frac{\omega_{c_{i}} \omega_{B}}{\omega_{e}} d_{e} \\
& =\sum_{e}\left\langle c_{i} B, e\right\rangle \frac{\omega_{c_{i}} \omega_{B}}{\omega_{e}} d_{e}=Y_{c_{i} B} .
\end{aligned}
$$

Q.E.D.

Recall the basis of $V_{0}$ corresponds to a finite set of irreducible covariant sectors of the coset: it is closed under composition, conjugation and contains identity by Th. 4.3 of $[\mathrm{X} 4]$. Define the $Y$ matrix as in (0) of $\S 2.1$. Then we have:

Theorem 2.3. (1) The matrix $Y$ is invertible. 
(2) The number $\tilde{\sigma}:=\Sigma_{e \in V} d_{e}^{2} \omega_{e}^{-1}$ is given by

$$
\tilde{\sigma}=\frac{1}{N} S_{11}^{-1} S_{11}^{-1} \exp \left(\frac{-6 \pi i}{24}\left(C_{G}-C_{H}\right)\right)
$$

where

$$
\frac{1}{24}\left(C_{G}-C_{H}\right)=\frac{N^{2}-1}{24}-\frac{N\left(N^{2}-1\right)}{24}\left(\frac{1}{m^{\prime}+N}+\frac{1}{m^{\prime}+N}-\frac{1}{m^{\prime}+m^{\prime \prime}+N}\right)
$$

Proof. By (i) of Prop. on P. 351 of [Reh] it is enough to show that if $e$ is such that

$$
Y_{e g}=d_{e} d_{g}, \quad \forall g
$$

then $e=1$ (Remember if a sector is denoted by 1 , then it is the identity or equivalently vacuum sector). Suppose $B:=P(j \otimes \beta)=\Sigma_{1 \leq i \leq t_{B}} g_{i} \succ g, B \in V_{0}$. By (4) of Lemma 2.2

$$
Y_{e B}=\sum_{i} Y_{e g_{i}}=\sum_{i} d_{e} d_{g_{i}}=d_{e} d_{B}
$$

Suppose $A=P(i \otimes \alpha)=\Sigma_{1 \leq i \leq t} c_{i} \succ e, d_{c_{i}}=\frac{1}{t} d_{c}$. Then by (3) of Lemma 2.2 we have:

$$
Y_{A B}=d_{A} d_{B}, \forall B \in V_{0}
$$

By (2) of Lemma 2.2 we have

$$
\frac{S_{i j}}{S_{11}} \frac{\bar{S}_{\alpha \beta}}{\dot{S}_{11}}=d_{i} d_{j} d_{\alpha} d_{\beta}
$$

and it follows that

$$
\frac{S_{i j}}{S_{1 j}} \frac{\dot{S}_{\alpha \beta}}{\dot{S}_{1 \beta}}=d_{i} d_{\alpha}
$$

for any $j \otimes \beta \in W_{0}$. Note

$$
\left|\frac{S_{i j}}{S_{1 j}}\right| \leq d_{i}, \quad\left|\frac{\dot{S}_{\alpha \beta}}{\dot{S}_{1 \beta}}\right| \leq d_{\alpha}
$$


there must exist $a_{i j} \in \boldsymbol{R}$ such that

$$
\frac{S_{i j}}{S_{1 j}}=\exp \left(i a_{i j}\right) d_{i}, \frac{\dot{S}_{\alpha \beta}}{\dot{S}_{1 \beta}}=\exp \left(i a_{i j}\right) d_{\alpha}
$$

for any $j \otimes \beta \in W_{0}$. Suppose $(j, \beta)=\left(\Lambda^{\prime}, \Lambda^{\prime \prime} ; \Lambda\right),(i, \alpha)=\left(M^{\prime}, M^{\prime \prime} ; M\right)$, then $\Lambda^{\prime}+\Lambda^{\prime \prime}$ $-\Lambda \in Q, M^{\prime}+M^{\prime \prime}-M \in Q$. From

$$
\frac{\dot{S}_{\alpha \beta}}{\dot{S}_{1 \beta}}=\exp \left(i a_{i j}\right) d_{\alpha}
$$

we get

$$
\left|\frac{S_{M \Lambda}}{S_{1 \Lambda}}\right|=d_{M}
$$

for any $\Lambda \in P_{+}^{\left(K^{\prime}+K^{\prime \prime}\right)}$, and so

$$
\sum_{\Lambda}\left|S_{M \Lambda}\right|^{2}=d_{M}^{2} \sum_{\Lambda}\left|S_{1 \Lambda}\right|^{2}
$$

i.e., $d_{M}^{2}=1$. It follows that $M \bar{M}$ is the identity sector, and so $M \Lambda$ is always irreducible. Choose $\Lambda$ corresponding to the defining representation of $S U(N)$, it follows from the fusion rules that $M$ must be of the form $\sigma^{s}(1)$ for some $s \in \mathbb{Z}$. Since $P(i \otimes \alpha)=P\left(\sigma^{-s}(i \otimes \alpha)\right)=A$, replacing $(i, \alpha)=\left(M^{\prime}, M^{\prime \prime} ; \sigma^{s}(1)\right)$ by $\sigma^{-s}(i$, $\alpha)=\left(\sigma^{-s}\left(M^{\prime}\right), \sigma^{-s}\left(M^{\prime \prime}\right) ; 1\right)$ if necessary, we may assume $s=0$, i.e., $\alpha=1$.

Similarly $\left(M^{\prime}, M^{\prime \prime}\right)=\left(\sigma^{s_{1}}(1), \sigma^{s_{2}}(1)\right)$, and using $\alpha=1$ we have

$$
\frac{S_{M^{\prime} \Lambda^{\prime}}}{S_{1 \Lambda^{\prime}}} \frac{S_{M^{\prime \prime} \Lambda^{\prime \prime}}}{S_{1 \Lambda^{\prime \prime}}}=d_{M^{\prime}} d_{M^{\prime \prime}}=1, \forall\left(\Lambda^{\prime}, \Lambda^{\prime \prime}\right)
$$

Choose $\Lambda^{\prime}$ to be the vaccum representation, from the above equation we have

$$
S_{M^{\prime \prime} \Lambda^{\prime \prime}}=S_{1 \Lambda^{\prime \prime}}, \forall \Lambda^{\prime \prime}
$$

Since $S$ matrix is unitary by $(9)$ in $\S 2.1$, we conclude $M^{\prime \prime}$ is the vacuum representation. By the same argument $M^{\prime}$ is also the vacuum representation.

So we have proved $P(i \otimes \alpha) \succ e$ is the vacuum sector, and therefore $e$ must be the vacuum sector.

Ad (2): First we claim:

$$
\sum_{e \in V_{0}} d_{e}^{2} \omega_{e}^{-1}=\frac{1}{N} \sum_{g \in W_{0}} d_{g}^{2} \omega_{g}^{-1} .
$$


Suppose $P(g)=f, P^{-1}(P(g))=\left\{g_{1}, \cdots, g_{n}\right\}$, and $P(g)=f=e_{1}+\cdots+e_{m}$, with $m n=N$. Since $\omega_{e_{i}}=\omega_{g}, d_{e_{i}}=\frac{d_{g}}{m}, d_{g_{j}}=d_{g}, \omega_{g_{j}}=\omega_{g}$, we have

$$
\sum_{e_{i}} d_{e_{i}}^{2} \omega_{e_{i}}^{-1}=\sum_{e_{i}} \frac{d_{g}^{2}}{m^{2}} \omega_{g}^{-1}=\frac{d_{g}^{2}}{m} \omega_{g}^{-1}
$$

and so

$$
\sum_{e_{i}} d_{e_{i}}^{2} \omega_{e_{i}}^{-1}=\frac{1}{N} n d_{g}^{2} \omega_{g}^{-1}=\frac{1}{N} \sum_{g_{j}} d_{g_{j}}^{2} \omega_{g_{j}}^{-1}
$$

It follows that

$$
\sum_{e \in V_{0}} d_{e}^{2} \omega_{e}^{-1}=\frac{1}{N} \sum_{g \in W_{0}} d_{g}^{2} \omega_{g}^{-1}
$$

Next let us show

$$
\sum_{g \in W_{1}} d_{g}^{2} \omega_{g}^{-1}=0
$$

Again Suppose $g=i \otimes \alpha, P(g)=f, P^{-1}(P(g))=\left\{g_{1}, \cdots, g_{n}\right\}$, and $P(g)=f=e_{1}+$ $\cdots+e_{m}$, with $m n=N$. So we have $\sigma^{n}(i, \alpha)=(i, \alpha), g_{k}=\sigma^{k-1} g_{1}, 1 \leq k \leq n$. Note that by definitions

$$
\omega_{\sigma(i)} \omega_{\sigma(\alpha)}^{-1}=\exp \left(\frac{-2 \pi i(\tau(i)-\tau(\alpha))}{N}\right) \omega_{i} \omega_{\alpha}^{-1}
$$

Denote by $z:=\exp \left(\frac{2 \pi i(\tau(i)-\tau(\alpha))}{N}\right)$, then $z^{n}=1$ since $\sigma^{n}(i, \alpha)=(i, \alpha)$, but $z \neq 1$ since $i \otimes \alpha$ is not in $W_{0}$, i.e., $\frac{(\tau(i)-\tau(\alpha))}{N} \notin Z$. So

$$
\sum_{g_{k}} d_{g_{k}}^{2} \omega_{g_{k}}^{-1}=\sum_{1 \leq k \leq n} d_{g}^{2} \omega_{g_{1}}^{-1} z^{k-1}=0
$$

We have 


$$
\begin{aligned}
\sum_{e \in V_{0}} d_{e}^{2} \omega_{e}^{-1} & =\frac{1}{N} \sum_{g \in W_{0}} d_{g}^{2} \omega_{g}^{-1}=\frac{1}{N} \sum_{g \in W} d_{g}^{2} \omega_{g}^{-1} \\
& =\frac{1}{N} \sum_{k} d_{k}^{2} \omega_{k}^{-1} \sum_{\delta} d_{\delta}^{2} \omega_{\delta} \\
& =\frac{1}{N} \exp \left(\frac{-6 \pi i\left(C_{G}-C_{H}\right)}{24}\right) \frac{1}{S_{11}} \frac{1}{S_{11}}
\end{aligned}
$$

where in the last $=$ we have used (3), (12) and comments after (12) in $\$ 2.1$, and $C_{G}$ and $C_{H}$ are central charges given by (1.4.2) of [KW], i.e.,

$$
\begin{gathered}
C_{G}=\frac{\left(N^{2}-1\right) m^{\prime}}{m^{\prime}+N}+\frac{\left(N^{2}-1\right) m^{\prime \prime}}{m^{\prime \prime}+N}, \\
C_{H}=\frac{\left(N^{2}-1\right)\left(m^{\prime}+m^{\prime \prime}\right)}{m^{\prime}+m^{\prime \prime}+N} .
\end{gathered}
$$

(2) now follows by a simple calculation.

Q.E.D.

Corollary 2.4. The irreducible covariant sectors of the diagonal coset of type A corresponding to the basis of $V_{0}$, with its braiding and $S, T$ matrices as defined in $\$ 2.1$, is a unitary modular category (cf. [Tu]).

Proof. By the definition of unitary modular category as on P. 74 and P. 113 of $[\mathrm{Tu}]$, it is enough to show that the $Y$ matrix is invertible, which follows from (1) of Th. 2.3.

Q.E.D.

When the $\mathbb{Z}$ action on $\exp$ is faithful, i.e., for any $(i, \alpha) \in \exp , \sigma^{s}(i, \alpha)=(i, \alpha)$ iff $N \mid s$, by (2) of Th. 2.3 we have that $S_{A B}=N S_{i j} \overline{S_{\alpha \beta}^{*}}$ where $A:=P(i \otimes \alpha)$, $B:=P(j \otimes \beta)$ and both $A, B$ are irreducible.

Let us calculate $S$-matrices in the case $N$ is a prime and there exists a (necessarily unique) fixed point $F:=P\left(i_{0} \otimes \alpha_{0}\right) \in V_{0}$ under the action of $\sigma$. By Lemma $2.1, F=\Sigma_{1 \leq i \leq N} F_{i}$ with irreducible and $d_{F_{i}}=\frac{1}{N} d_{F} . \quad$ Recall $S_{a b}:=|\tilde{\sigma}|^{-1} Y_{a b}$, where

$$
|\tilde{\sigma}|^{2}=\frac{1}{N^{2}} \frac{1}{S_{11}^{2}} \frac{1}{S_{11}^{2}}
$$

which follows from (2) of Th. 2.3. 
So $S_{a b}=N S_{11} S_{11} Y_{a b}$, and by (3) of Lemma $2.2 S_{a b}$ for $a=P(i \otimes \alpha) \neq F_{i}$ is determined as follows:

$$
S_{a b}=N S_{i j} \overline{S_{\alpha \beta}^{*}}
$$

if $b:=P(j \otimes \beta) \neq F_{k}$, and

$$
S_{a F_{k}}=S_{i i_{0}} \overline{S_{\alpha \alpha_{0}}^{\cdot}} .
$$

It remains to determine $S_{F_{1} F_{j}}$. Note

$$
S T S=T^{-1} S T^{-1}
$$

and $T_{a b}=\delta_{a b} \dot{\omega}_{a}$, where $\dot{\omega}_{a}=\omega_{a} \omega$, and $\omega$ is determined by (2) of Th. 2.3 and (3) of $\S 2.1$ as:

$$
\omega:=\exp \left(\frac{-2 \pi i}{24}\left(C_{G}-C_{H}\right)\right)
$$

We have

$$
\begin{aligned}
\omega_{F_{i}}^{-1} S_{F_{l} F_{k}} \omega_{F_{k}}^{-1} & =\sum_{a} S_{F_{l} A} \dot{\omega}_{A} S_{A F_{k}} \\
& =\sum_{l} S_{F_{i} F_{l}} \omega_{F_{l}} S_{a F_{k}}+\sum_{A \neq F} S_{F_{i} A} \omega_{A} S_{A F_{k}} \\
& =\omega_{F} \sum_{l} S_{F_{i} F_{l}} S_{F_{l} F_{k}}+\sum_{A \neq F} S_{F_{l} A} \omega_{A} S_{A F_{k}} \\
& =\omega_{F}\left(\delta_{F_{i} \bar{F}_{k}}-\sum_{B \neq F} S_{F_{i} B} S_{B F_{k}}\right)+\sum_{A \neq F} S_{F_{i} A} \omega_{A}^{\cdot} S_{A F_{k}} \\
& =\omega_{F} \delta_{F_{i} \bar{F}_{k}}+\sum_{A \neq F} S_{F_{i} A}\left(\omega_{A}^{\cdot}-\omega_{F}\right) S_{A F_{k}} \\
& =\omega_{F} \delta_{F_{i} \bar{F}_{k}}+\frac{1}{N^{2}} \sum_{A \neq F} S_{F A}\left(\omega_{A}^{\cdot}-\omega_{F}\right) S_{A F} \\
& =\omega_{F} \delta_{F_{i} \bar{F}_{k}}+\frac{1}{N^{2}} \sum_{A} S_{F A}\left(\omega_{A}^{\cdot}-\omega_{F}\right) S_{A F}
\end{aligned}
$$

where we used $S^{2}$ is equal to conjugate matrix in the fourth $=$, and (3) of Lemma 2.2 in the sixth $=$. So we have 


$$
S_{F_{i} F_{k}}=\omega_{F}^{3}+\omega_{F}^{2} \frac{1}{N^{2}} \sum_{A \in V_{0}} S_{F A}\left(\omega_{A}^{\cdot}-\omega_{F}^{*}\right) S_{A F}
$$

But

$$
\begin{aligned}
\sum_{A \in V_{0}} S_{F A}\left(\omega_{A}-\omega_{F}\right) S_{A F} & =\sum_{A \in V_{0}, 1 \leq i, j \leq N} S_{F_{i} A}\left(\omega_{A}-\omega_{F}\right) S_{A F_{j}} \\
& =\sum_{A \in V_{0}, 1 \leq i, j \leq N} S_{F_{i} A} \omega_{A}^{\cdot} S_{A F_{j}}-\sum_{A \in V_{0}, 1 \leq i, j \leq N} S_{F_{i} A} \omega_{F} S_{A F_{j}} \\
& =\sum_{1 \leq i, j \leq N} \omega_{F}^{-2} S_{F_{i} F_{j}}-\sum_{A \in V_{0}, 1 \leq i, j \leq N} S_{F_{i} A} \omega_{F} S_{A F_{j}} \\
& =\omega_{F}^{-2} S_{F F}-\omega_{F}^{\cdot} \sum_{1 \leq i, j \leq N} \delta_{F_{i} \bar{F}_{j}} \\
& =\omega_{F}^{-2} S_{F F}-\omega_{F} N
\end{aligned}
$$

where $S_{F F}=|\tilde{\sigma}|^{-1} Y_{F F}$, and in the fourth = we used (4) of Lemma 2.2, and in the last step we used $\bar{F}=F$ and

$$
\sum_{1 \leq i, j \leq N} \delta_{F_{i} \bar{F}_{j}}=\langle F, \bar{F}\rangle=\langle F, F\rangle=N
$$

So we have:

$$
S_{F_{i} F_{k}}=\omega_{F}^{3} \delta_{F_{i} \bar{F}_{k}}+\frac{1}{N^{2}}\left(S_{F F}-\omega_{F}^{3} N\right)
$$

Let us show

$$
\omega_{F}^{3}=\omega_{F}^{3} \omega^{3}=1
$$

Recall $\omega_{F}=\exp \left(2 \pi i \Delta_{F}\right)$, and since $F$ is the unique fixed point, by a simple calculation using (10) of $\S 2.1$ we get

$$
\Delta_{F}=\frac{N^{2}-1}{24}-\frac{N\left(N^{2}-1\right)}{24}\left(\frac{1}{m^{\prime}+N}+\frac{1}{m^{\prime}+N}-\frac{1}{m^{\prime}+m^{\prime \prime}+N}\right),
$$

and it follows that

$$
\omega=\exp \left(-2 \pi i \frac{1}{24}\left(C_{G}-C_{H}\right)\right)=\omega_{F}^{-1}
$$


by (2) of Th. 2.3 , so $\omega_{F}=1$.

Therefore we have

$$
S_{F_{i} F_{k}}=\delta_{F_{i} \bar{F}_{k}}+\frac{1}{N^{2}}\left(S_{F F}-N\right) .
$$

Since $\bar{F}=F, S_{F A}$ is real for all $A$, so $S_{F_{k} a}$ is real for any irreducible $a$, and we must have $\bar{F}_{k}=F_{k}$ since $S$ matrix is invertible. So:

$$
S_{F_{i} F_{k}}=\delta_{i k}+\frac{1}{N^{2}}\left(S_{F F}-N\right)
$$

The formula (2), (3) and (4) above agree wiht formula (4.40) of [FSS1] (Note our $S_{F F}=N S_{i_{0} i_{0}} \overline{S_{\alpha_{0} \alpha_{0}}^{-}}$, where $\left.F=P\left(i_{0} \otimes \alpha_{0}\right)\right)$. However one should notice that our definition of $S$ matrices are very different from those of [FSS1].

In [FSS1] and [FSS2], certain formula about $\mathrm{S}$ matrices were derived from other considerations in the case when $N$ is not prime and other types of simple simply connected Lie groups, and it will be interesting to extend our calculations above to these cases and to see if the results agree with [FSS1] and [FSS2].

By Cor. 2.4, one may calculate 3-manifold invariants using $S$ matrices obtained above as in [Tu]. These and related questions will be addressed in another publication.

\section{§3. Miscellaneous Results}

§3.1 KWH and KWC. Let $H \subset G_{k}$ be as in the introduction. Through out this section, we will assume the following: $H$ and $G$ verifies the statements as in Cor. 1 in $\S 34$ of [W] (cf: comments after (12) in $\S 2.1$ ), and $H \subset G_{k}$ is cofinite as defined in $\S 3$ of $[\mathrm{X} 4]$.

Note the assumption is satisfied by many examples (cf. Cor. 4.2 of [X4]) and is expected to be true in general.

We also assume that $H \subset G_{k}$ is not conformal, so the coset theory is non-trival.

We will use the notations of $\S 4.2$ of [X4] and ideas of [X2]. We denote the set of irreducible sectors of $\sigma_{i} a_{1 \otimes \lambda}$ by $V$. Notice $\sigma_{i} \in V$, and these are referred to as special nodes in $\S 3.4$ of [X1]. Let:

$$
a_{1 \otimes \lambda} a=\sum_{b \in V} V_{a b}^{\lambda} b
$$


where $V_{a b}^{\lambda}$ are nonnegative integers. Denote by $V^{\lambda}$ the matrix such that $\left(V^{\lambda}\right)_{a}^{b}=V_{a b}^{\lambda}$. Define matrix $N_{c}$ by $N_{c a}^{b}=\langle c a, b\rangle$ for $a, b, c \in V$. Then $V^{\lambda}$ $=\Sigma_{c} V_{1 c}^{\lambda} N_{c}$. Since $\left[a_{1 \otimes \bar{\lambda}}\right]=\left[\bar{a}_{1 \otimes \lambda}\right],\left[\sigma_{j} a_{1 \otimes \lambda}\right]=\left[a_{1 \otimes \lambda} \sigma_{j}\right], V^{\lambda}, N_{\sigma_{j}}$ are commuting normal matrices, so they can be simultaneously diagonalized. Recall the irreducible representations of the ring $\operatorname{Gr}\left(C_{k}\right)$ generated by $\lambda^{\prime} s$ are given by

$$
\lambda \rightarrow \frac{S_{\lambda \mu}}{S_{1 \mu}}
$$

Assume

$$
V_{a b}^{\lambda}=\sum_{i, \mu, s \in(\mathbf{E x p})} \frac{S_{\lambda \mu}}{S_{1 \mu}} \psi_{a}^{(i, \mu, s)} \psi_{b}^{(i, \mu, s) *}
$$

where $\psi_{a}^{(i, \mu, s)}$ are normalized orthogonal eigenvectors of $V^{\lambda}$ (resp. $N_{\sigma_{1}}$ ) with eigenvalue $\frac{S_{\lambda \mu}}{S_{1 \mu}}\left(\right.$ resp. $\frac{S_{i j}}{S_{1 j}}$ ). (Exp) is a set of $i, \mu, s^{\prime}$ s and $s$ is an index indicating the multiplicity of $i, \mu$. We denote by $\operatorname{Exp}$ the set of $(i, \mu)$ such that $(i, \mu, s) \in(\operatorname{Exp})$ for some $s$. Recall if a representation is denoted by 1 , it will always be the vacuum representation. The Perron-Frobenius eigenvector $\psi^{(1,1)}$ is given by $\Sigma_{a} d_{a} a$, up to a positive constant. Note all the entries of $\psi^{(1,1)}$ are positive.

Proposition 3.1. $(i, \alpha) \in$ Exp if and only if $b(i, \alpha)>0$.

Proof. Recall (1) of $\S 1$ :

$$
b(i, \alpha)=\sum_{(j, \beta)} S_{i j} \dot{S}_{\alpha \beta}\langle(i, \alpha),(1,1)\rangle .
$$

By the proof of (2) of Prop. 4.2 of [X4] and (2) of Cor. 3.5 of [X1] we have

$$
\langle(i, \alpha),(1,1)\rangle=\left\langle\sigma_{i} a_{1 \otimes \bar{\alpha}}, 1\right\rangle=\left\langle\sigma_{i} a_{1 \otimes \alpha}, 1\right\rangle,
$$

so:

$$
b(i, \alpha)=\sum_{(j, \beta)} S_{i j} \dot{S}_{\alpha \beta}\left\langle\sigma_{\bar{j}} a_{1 \otimes \beta}, 1\right\rangle=\sum_{(j, \beta)} S_{i j} \dot{S}_{\alpha \beta}\left(N_{\bar{j}} V^{\beta}\right)_{11}
$$




$$
\begin{aligned}
& =\sum_{(k, \delta, s) \in(E x p)} S_{i j} \dot{S}_{\alpha \beta} \frac{\dot{S}_{\beta \delta}}{\dot{S}_{1 \delta}} \frac{\overline{S_{j k}}}{S_{1 k}}\left|\psi_{1}^{(k, \delta, s)}\right|^{2} \\
& =\sum_{s} \frac{1}{\dot{S}_{1 \alpha}} \frac{1}{S_{1 i}}\left|\psi_{1}^{(i, \alpha, s)}\right|^{2} .
\end{aligned}
$$

Note the equality above is similar to (1) on P. 12 of [X2], and the rest of the proof is the same as the proof on P. 12 of [X2].

Q.E.D.

Note if $b(i, \alpha)>0$, then $(i, \alpha) \in \exp$, so by Prop. $3.1 \operatorname{Exp} \subset \exp$, and KWC is equivalent to the statement that $\operatorname{Exp}=\exp$.

Proposition 3.2. If $b(i, 1)>0$, then $\frac{b(i, 1)}{b(1,1)}=\frac{S_{i 1}}{S_{11}}$.

Proof. If $b(i, 1)>0$, by Prop. 3.1, $(i, 1) \in$ Exp. Suppose $(j, \beta)>(1,1) . \quad$ Then

$$
\langle(j, \beta),(1,1)\rangle=\left\langle\sigma_{j} a_{1 \otimes \bar{\beta}}, 1\right\rangle=\left\langle\sigma_{j}, a_{1 \otimes \beta}\right\rangle>0
$$

since $\sigma_{j}$ is irreducible. It follows that

$$
a_{1 \otimes \beta} \succ \sigma_{j},
$$

and if $b=a_{1 \otimes \beta}-\sigma_{j}$, then $V^{b}:=V^{\beta}-N_{j}$ is a normal matrix with non-negative entries, with a Perron-Frobenius eigenvalue $\frac{\dot{\boldsymbol{S}}_{\beta 1}}{\dot{\boldsymbol{S}}_{11}}-\frac{\boldsymbol{S}_{j 1}}{\boldsymbol{S}_{11}}$. It follows that

$$
\begin{aligned}
\frac{\dot{S}_{\beta 1}}{\dot{S}_{11}}-\frac{S_{j 1}}{S_{11}} & \geq\left|\left\langle V^{b} \psi^{(i, 1, s)}, \psi^{(i, 1, s)}\right\rangle\right|=\left|\frac{\dot{S}_{\beta 1}}{\dot{S}_{11}}-\frac{S_{j i}}{S_{1 i}}\right| \\
& \geq \frac{\dot{S}_{\beta 1}}{\dot{S}_{11}}-\left|\frac{S_{j i}}{S_{1 i}}\right| \\
& \geq \frac{\dot{S}_{\beta 1}}{\dot{S}_{11}}-\frac{S_{j 1}}{S_{11}} .
\end{aligned}
$$

So all the $\geq$ are $=$, which happens only if

$$
\frac{S_{j i}}{S_{1 i}}=\frac{S_{j 1}}{S_{11}} .
$$

Prop. 3.1 now follows from the definitions.

Q.E.D.

Theorem 3.3. If $H \subset G_{k}$ satisfies (2) in the introduction, then $K W H$ is 
equivalent to $K W C$.

Proof. We just have to show that KWC implies KWH.

By (2) of the introduction we have if $\langle(1, \delta),(1,1)\rangle>0$, then $\delta=1$. For any $\alpha, \beta$, we have (cf. Prop. 4.2 of [X4]):

$$
\begin{aligned}
\left\langle a_{1 \otimes \alpha}, a_{1 \otimes \beta}\right\rangle=\left\langle a_{1 \otimes \alpha} a_{1 \otimes \bar{\beta}}, 1\right\rangle & =N_{\alpha \bar{\beta}}^{\delta}\left\langle a_{1 \otimes \delta}, 1\right\rangle \\
& =N_{\alpha \bar{\beta}}^{\delta}\langle(1, \delta),(1,1)\rangle \\
& =N_{\alpha \bar{\beta}}^{1} \\
& =\langle\alpha, \beta\rangle .
\end{aligned}
$$

In particular $a_{1 \otimes \beta}$ is irreducible if $\beta$ is irreducible.

Suppose $\langle(j, \beta),(1,1)\rangle>0$, then $\left\langle\sigma_{j} a_{1 \otimes \bar{\beta}}, 1\right\rangle>0$, and so $\left\langle\sigma_{j}, a_{1 \otimes \beta}\right\rangle>0$. Since both $\sigma_{j}$ and $a_{1 \otimes \beta}$ are irreducible, it follows that

$$
\sigma_{j}=a_{1 \otimes \beta} .
$$

Now suppose $(i, \alpha) \in \exp$. By KWC, $b(i, \alpha)>0$, so by Prop. 3.1, $(i, \alpha) \in \operatorname{Exp}$, and from $\sigma_{j}=a_{1 \otimes \beta}$ we must have:

$$
\frac{S_{j i}}{S_{1 i}}=\frac{\dot{S}_{\beta \alpha}}{\dot{S}_{1 \alpha}} .
$$

Therefore

$$
S_{j i} \bar{S}_{\beta \alpha}=S_{1 i} \frac{\left|\dot{S}_{\beta \alpha}\right|^{2}}{\dot{S}_{1 \alpha}} \geq 0
$$

which proves $\mathrm{KWH}$.

Q.E.D.

Let us give an example which does not satisfy the assumption of our theorem, and verifies $\mathrm{KWC}$ but not KWH. This is the coset $S U(2)_{8} \subset S U(3)_{2}$ discussed in $\S 4.4$ of [X4] (also cf. [DJ]) and we will use the notations there. The vacuum representation space $H$ of $L S U(3)$ at level 2 decomposes as:

$$
H=(00,0) \otimes 0+(00,4) \otimes 4+(00,8) \otimes 8,
$$

and since

$$
(00,0)=(00,8)
$$

as representations of the coset, our assumption is not satisfied. In $\S 4.4$ of [X4] 
we checked $\mathrm{C} 2$ is satisfied, which implies that $\mathrm{KWC}$ is true. However, since

$$
(11,4)=(00,0)
$$

and $(10,4) \in \exp , \mathrm{KWH}$ implies that

$$
S_{11,10} \dot{S}_{4,4} \geq 0
$$

But a direct calculation using (9) of $\$ 2.1$ gives

$$
\frac{S_{11,10}}{S_{00,10}}=\frac{1-\sqrt{5}}{2}
$$

and

$$
S_{4,4}=\sqrt{\frac{1}{5}},
$$

which shows that

$$
S_{11,10} S_{4,4}^{\cdot<0}
$$

since $S_{00,10}>0$. In fact this was discovered when we verified $\mathrm{C} 2$ in this example.

Note that all diagonal inclusions of type $A$ satisfies the assumption of Th. 3.3 by 2.7 .12 of [KW]. To give a slightly different example, let us consider the following inclusions

$$
S U(2)_{11 k} \subset S U(2)_{8 k} \times S U(2)_{3 k} \subset S U(3)_{2 k} \times S U(2)_{3 k} \subset S U(6)_{k}
$$

with $k \in N$, where the first inclusion is diagonal, the second inclusion comes from the conformal inclusion $S U(2)_{4} \subset S U(3)_{1}$, and the third inclusion comes from the conformal inclusion $S U(3)_{2} \times S U(2)_{3} \subset S U(6)$ (cf. [X3]).

By (2) of Prop. 3.1 and (2) of Cor. 3.1 of [X4], the inclusion $S U(2)_{11 k} \subset$ $S U(6)_{k}$ is cofinite and verifies the assumptions at the beginning of this section. Note if $(1, \alpha) \succ(1,1)$, then $\Delta_{\alpha} \in Z$ by definition. Here $1 \leq \alpha \in Z \leq 11 k+1$ and $\Delta_{\alpha}=\frac{\alpha(\alpha-1)}{11 k+2}$. So if $11 k+2$ is prime, then $(1, \alpha) \succ(1,1)$ iff $\alpha=1$, and it follows that the conditions of Th. 2.3 are satisfied. So the conclusions of Th. 2.3 hold for the inclusion

$$
S U(2)_{11_{k}} \subset S U(6)_{k}
$$

if $11 k+2$ is prime, and by Dirichlet's theorem, there are infinitely many such $k$ 's. 


\section{§3.2. A property of $\mathbf{C} 2$.}

Proposition 3.4. If $H_{1} \subset H_{2}$ (resp. $H_{2} \subset G_{k}$ ) verifies $C 2$ or is a conformal inclusion, and assume $H_{1} \subset G_{k}$ is not a conformal inclusion to avoid trivality, then $H_{1} \subset G_{k}$ verifies $C 2$.

Proof. For simplicity we will use $\pi_{x}, \pi_{y}, \pi_{z}$ to denote the irreducible representations of $L H_{1}, L H_{2}$ and $L G$ respectively, and $\mathscr{A}, \mathscr{B}, \mathscr{C}$ to denote the vacuum sector of cosets $H_{1} \subset H_{2}, H_{2} \subset G, H_{1} \subset G$ respectively. Note we have natural inclusions $\mathscr{A}(I) \otimes \mathscr{B}(I) \subset \mathscr{C}(I)$, corresponding to the natural inclusions

$$
\left(\pi\left(L_{1} H_{1}\right)^{\prime} \cap \pi\left(L_{I} H_{2}\right)^{\prime \prime}\right) \otimes\left(\pi\left(L_{I} H_{2}\right)^{\prime} \cap \pi\left(L_{I} G\right)^{\prime \prime}\right) \subset \pi\left(L_{I} H_{1}\right)^{\prime} \cap \pi\left(L_{I} G\right)^{\prime \prime},
$$

where $I$ is a proper open interval of $S^{1}$. From the decompositions:

$$
\pi_{z} \simeq \sum_{y} \pi_{(z, y)} \otimes \pi_{y} \simeq \sum_{y, x} \pi_{(z, y)} \otimes \pi_{(y, x)} \otimes \pi_{x} \simeq \sum_{x} \pi_{(z, x)} \otimes \pi_{x}
$$

we conclude that

$$
\pi_{(z, x)} \simeq \sum_{y} \pi_{(z, y)} \otimes \pi_{(y, x)}
$$

which is understood as the decomposition of representation $\pi_{(z, x)}$ of $C$ when restricted to $A \otimes B \subset C$. By local equivalence (cf. P. 502 of [W]), the minimal index of

$$
\pi_{(z, x)}(A(I) \otimes B(I)) \subset \pi_{(z, x)}(C(I))
$$

is the same as that of

$$
\pi_{(1,1)}(A(I) \otimes B(I)) \subset \pi_{(1,1)}(C(I))
$$

( $I$ is a proper interval of the circle), which by Haag duality (cf. Prop. 1.1 of [GL]) and Th. 5.5 of [L1] is given by

$$
\sum_{y} d_{(1, y)} d_{(y, 1)}
$$

Here when $H_{2} \subset G$ (resp. $H_{1} \subset H_{2}$ ) is a conformal inclusion, $d_{(z, y)}\left(\right.$ resp. $\left.d_{(y, x)}\right)$ is defined to be the multiplicity of irreducible representation $y$ (resp. $x$ ) which 
appears in $z$ (resp. $y$ ) when restricting to $L H_{2}$ (resp. $L H_{1}$ ). Otherwise $d_{(z, y)}$ (resp. $\left.d_{(y, x)}\right)$ is the statistical dimension (cf. §2.1) of sector $(z, y)$ (resp. $(y, x)$ ).

By Cor. 2.2 of [L3], the statistical dimension of the inclusion: $\pi_{(z, x)}(A(I) \otimes B(I)) \subset \pi_{(z, x)}(C(I)) \subset \pi_{(z, x)}\left(C\left(I^{\prime}\right)\right)^{\prime} \subset \pi_{(z, x)}\left(A\left(I^{\prime}\right) \otimes B\left(I^{\prime}\right)\right)^{\prime}\left(I^{\prime}\right.$ is the complement of $I$ in $S^{1}$ as defined on P. 14 of [GL]) is

$$
d_{(z, x)} \sum_{y} d_{(1, y)} d_{(y, 1)}
$$

On the other hand, by Th. 5.5 of [L1], the statistical dimension of the above inclusion is also given by:

$$
\sum_{y} d_{(z, y)} d_{(y, x)}
$$

and so:

$$
d_{(z, x)} \sum_{y} d_{(1, y)} d_{(y, 1)}=\sum_{y} d_{(z, y)} d_{(y, x)}
$$

Therefore

$$
d_{(z, x)}=\frac{\Sigma_{y} d_{(z, y)} d_{(y, x)}}{\Sigma_{y} d_{(1, y)} d_{(y, 1)}}
$$

The proof now follows from the assumptions and

$$
b(z, x)=\sum_{y} b(z, y) b(y, x)
$$

which follows from Th. B of [KW].

Q.E.D.

Let us give one application of the above proposition. Consider the superconformal coset models (cf. [Gep], [LVW] or [NS]):

$$
G(m, n, k):=\frac{S U(m+n)_{k} \times S O(2 m n)_{1}}{S U(m)_{n+k} \times S U(n)_{m+k} \times U(1)_{m n(m+n)(m+n+k)}} .
$$

In our setting, when $2 m n>2$, the inclusion is given by $H \subset G$ with $H=S U(m)_{n+k} \times S U(n)_{m+k} \times U(1)_{m n(m+n)(m+n+k)}$ and $G=S U(m+n)_{k} \times \operatorname{Spin}(2 m n)_{1}$. The inclusion $H \subset G$ is constructed by the composition of two inclusions: 


$$
\begin{gathered}
H \subset S U(m)_{n} \times S U(m)_{k} \times S U(n)_{m} \times S U(n)_{k} \\
\times U(1)_{m n(m+n)(m+n)} \times U(1)_{m n(m+n)(k)}
\end{gathered}
$$

and

$$
\begin{aligned}
\left(S U(m)_{n} \times\right. & \left.S U(n)_{m} \times U(1)_{m n(m+n)(m+n)}\right) \times\left(S U(m)_{k} \times S U(n)_{k}\right. \\
& \left.\times U(1)_{m n(m+n)(k)}\right) \subset S p i n(2 m n)_{1} \times S U(m+n)_{k} .
\end{aligned}
$$

The tangent space of the Grassmanian

$$
\frac{S U(m+n)}{S U(m) \times S U(n) \times U(1)}
$$

at the point corresponding to the identity of $S U(m+n)$ is isomorphic to $\mathbb{C}^{m} \otimes \mathbb{C}^{n}$, which is a fundamental representation of $\operatorname{Spin}(2 m n)$. The natural action of $S U(m) \times S U(n) \times U(1)$ on the tangent space gives the conformal inclusion (cf. $\S 4.2$ of $[\mathrm{KW}])$

$$
S U(m)_{n} \times S U(n)_{m} \times U(1)_{m n(m+n)(m+n)} \subset S p i n(2 m n)_{1} .
$$

The inclusion

$$
S U(m)_{k} \times S U(n)_{k} \times U(1)_{m n(m+n)(k)} \subset S U(m+n)_{k}
$$

comes from the conformal inclusion (cf. Prop. 4.2 of $[\mathrm{KW}]$ )

$$
S U(m)_{1} \times S U(n)_{1} \times U(1)_{m n(m+n)} \subset S U(m+n)_{1} .
$$

The inclusion in (1) is diagonal, and the $S U$ part of the inclusion verifies $\mathrm{C} 2$ by (4) of Th. 4.3 in [X4]. For the $U(1)$ part, we consider the following inclusions:

$$
U(1)_{2 a} \times U(1)_{2 b} \subset S U(2)_{a} \times S U(2)_{b},
$$

and

$$
U(1)_{2 a+2 b} \subset S U(2)_{a+b} \subset S U(2)_{a} \times S U(2)_{b},
$$

with $a:=\frac{1}{2} m n(m+n)^{2}, b:=\frac{1}{2} m n(m+n) k$. It follows from (3) of Cor. 3.1 of [X4] and the proof of (1) of Prop. 3.1 that

$$
U(1)_{2 a+2 b} \subset U(1)_{2 a} \times U(1)_{2 b}
$$

is cofinite, and since all the endomorphisms involved are automorphisms as 
in the paragraph after lemma 3.2 of [X4], C2 is immediately verified in this case.

It follows from Prop. 3.1 and Th. 4.2 of [X4], [W] and [B] that $G(m, n, k)$ coset verifies Conj. 1 of [X4], and so is indeed a "rational" conformal field theory.

By Proposition 3.2, we see that when $k=1$ and $m n>1$, the above coset verifies $\mathbb{C} 2$.

The fixed point resolution problems for $G(m, n, k)$ are discussed in $[\mathrm{Gep}]$, [LVW] (also cf. [NS]). It will be interesting to work out this problem along the lines of $\S 2$.

\section{References}

[B] Böckenhauer, J., An algebraic formulation of level $1 \mathrm{WZW}$ models, Rev. Math. Phys., 8 (1996), 925-948.

[DJ] Dunbar, D. and Joshi, K., Characters for coset conformal field theories and maverick examples, Inter. J. Mod. Phys. A, \&, No. 23 (1993), 4103-4121.

[Dyn1] Dynkin, E. B., Semisimple subalgebras of semisimple Lie algebras, Amer. Math. Soc. Transl. (2), 6 (1957), 111-245.

[Dyn2] - Maximal subgroups of classical groups, Amer. Math. Soc. Transl. (2), 6 (1957), 245-379.

[FRS] Fredenhagen, K., Rehren, K.-H. and Schroer, B., Superselection sectors with braid group statistics and exchange algebras II, Rev. Math. Phys., Special issue (1992), 113-157.

[FSS1] Fuchs, J., Schellekens, B. and Schweigert, C., The resolution of field identification fixed points in diagonal coset theories, Nucl. Phys. B, 461 (1996), 371.

[FSS2] , From Dynkin diagram symmetries to fixed point structures, Comm Math. Phys., 180 (1996), 39.

[Gep] Gepner, D., Field identifications in coset conformal field theories, Phys. Lett. B, 222 (1989), 207.

[GL] Guido, D. and Longo, R., An Algebraic Spin and Statistics Theorem, Comm. Math. Phys., 181 (1996), 11-35.

[J] Jones, V., Fusion en algèbres de Von Neumann et groupes de lacets (d'après A. Wassermann), Seminarie Bourbaki, 800 (1995), 1-20.

[KW] Kac, V. G. and Wakimoto, M., Modular and conformal invariance constraints in representation theory of affine algebras, Adv. Math., 70 (1988), 156-234.

[Kac] Kac, V. G., Infinite dimensional Lie algebras, 3rd Edition, Cambridge University Press, 1990.

[Ka] Kawahigashi, Y., Classification of paragroup actions on subfactors, Publ. RIMS, Kyoto Univ., 31 (1995), 481-517.

[LVW] Lerche, W., Vafa, C. and Warner, N. P., Nucl. Phys. B, 324 (1989), 427.

[L1] Longo, R., Index of subfactors and statistics of quantum fields, I, Comm. Math. Phys., 126 (1989), 217-247.

[L2] - Index of subfactors and statistics of quantum fields, II, Comm. Math. Phys., 130 (1990), 285-309.

[L3] - Minimal index and braided subfactors, J. Funct. Anal., 109 (1992), 98-112.

[L4] An analog of the Kac-Wakimoto formula and black hole conditional entropy, Comm. Math. Phys., 186 (1997), 451-479.

[LR] Longo, R. and Rehren, K.-H., Nets of subfactors, Rev. Math. Phys., 7 (1995), 567-597.

[NS] Naculich, S. and Schnitzer, H., Superconformal coset equivalence from levelrank duality, hep-th/9705149.

[PP] Pimsner, M. and Popa, S., Entropy and index for subfactors, Ann. Ec. Norm. Sup., 
19 (1986), 57-106.

[PS] Pressly, A. and Segal, G., Loop Groups, O.U.P. 1986.

[Reh] Rehren, K.-H., Braid group statistics and their superselection rules, The algebraic theory of superselection sectors. World Scientific 1990.

[SY] Schellekens, A. N. and Yankielowicz, S., Field identification fixed points in the coset construction, Nucl. Phys. B, 324 (1990), 67.

[Tu] Turaev, V. G., Quantum invariants of knots and 3-manifolds, Walter de Gruyter, Berlin, New York 1994.

[W] Wassermann, A., Operator algebras and conformal field theories III, Invent. Math., 133 (1998), 467-539.

[X1] Xu, F., New braided endomorphisms from conformal inclusions, Comm. Math. Phys., 192 (1998), 349-403.

[X2] - Applications of braided endomorphisms from conformal inclusions, Inter. Math. Res. Notice., No.1, (1998), 5-23, see also q-alg/9708013, and Erratum, Inter. Math. Res. Notice., No. 8, (1998).

[X3] - Jones-Wassermann subfactors for disconnected intervals, q-alg/9704003.

[X4] - Algebraic coset conformal field theories, q-alg/9810053. 